\title{
The evidence for the incorporation of fulvic acid into the bone and cartilage of rats
}

\author{
Wang Chunxia*, Wang Zijian, Yang Chunlin, Wang Wenhua, Peng An \\ State Key Laboratory of Environmental Aquatic Chemistry. Research Center for Eco-Entironmental Sciences, Academia Sinica, PO \\ Box 2871, Beijing 100085, People's Rebublic of China
}

Received 5 April 1996; accepted 28 June 1996

\begin{abstract}
In animal testing. abdominal injection of commercial fulvic acid (FA) to Wistar rats resulted in the coloration of the bone. Free radical signal was detected by ESR spectra of the bone, which were shown to be dependent upon the dosage of FA injected. The fluorescence spectra. UV spectra and gel permeation chromatography of the alkaline extracts of the bone showed similar characteristics to FA. In in-vivo rat tests and in-vitro experiments of cultured chicken cartilage tissue with tritium labeled FA, the results showed that ${ }^{3} \mathrm{H}-\mathrm{FA}$ could be incorporated into the bone and cartilage of rats.
\end{abstract}

Ke!words: Bone; Cartilage; Fulvic acid; Incorporation; Kaschin-Beck disease

\section{Introduction}

Kaschin-Beck disease (KBD) is regarded as an endemic, chronic, high incidence and degenerative osteoarthropathic disease. In China, an estimation of KBD patients in 1985 indicated that more than 2 million people were severely affected in a broad region inhabited by over 30 million people. KBD endemic areas form a broad belt extending from northeast to southwest which is the low selenium belt in China. The disease mostly appears in

\footnotetext{
* Corresponding author.
}

mountainous and hilly regions, and farmers are mainly affected. In the etiologic study for the cause of KBD, it has been suggested that fulvic acid (FA) in potable water is one of the environmental factors (Peng et al., 1991). Humic substances account for $85-90 \%$ of organic matters in soil and ground water (Aiken, 1985) and FA in the soluble fraction. The previous works have found that FA from potable water of KBD prone regions could cause damage to the chondrocyte and result in a decrease of the activity of glutathione peroxidase (GSH-Px) in in-vitro cartilage culture (Peng et al., 1991). On the other hand, FA 
can cause an increase of the lipid peroxides in the liver and blood of rats in-vivo (Feng and Wang, 1990). In the survey of Blackfoot Disease (Lu et al., 1980) and thyromegaly (Huang et al., 1993), it was suggested that FA might be one of the causative factors. FA is a macromolecule with molecular weight ranging from several thousands to many thousands and is versatile in the environment. While previous studies showed that FA could be a potential factor related to human health, knowledge of its incorporation into mammal tissue remains unclear. Regarding the etiology, the causative factor or toxicant should reach directly or indirectly the target tissue before its action. KBD is characterized by pathological clinical changes of a limb's articular function. The epiphyseal plate cartilage, bone and joint cartilage are the target tissues. As a causative factor of KBD or other FA related skeleton diseases, FA incorporation into bone and cartilage needs to be verified. In this study, FA from peat and tritium labeled FA from a $\mathrm{KBD}$ prone area were used in the investigation of $\mathrm{FA}$ incorporation into mammal tissues.

\section{Materials and methods}

\subsection{Accumulation of peat $F A$ in the bone of rats}

Purchased peat FA (Beijing FA) was dissolved in distilled water and adjusted to $\mathrm{pH} 7.4$ using $\mathrm{NaOH}$ solution. The prepared FA solution was made isotonic by adding $\mathrm{NaCI}$ and then sterilized. The Wistar rats $(150-180 \mathrm{~g})$ with equal numbers of female and malc were divided into two groups of 20 . FA solution was abdominally injected into the rats over a period of 130 days. Three dosage levels were administrated of 50 . 100 and $150 \mathrm{mg}$ FA $/ \mathrm{kg}$ per day, respectively. The rats in the control group were injected with physiological saline. After 130 days, the rats were sacrificed and the four limb bones were separated. The specimens were preserved in formalin.

\subsection{Measurement of ESR spectra for bone specimens}

The bone preserved in formalin was cleaned with distilled water and dried under an infra-red lamp. The specimen was minced and put into a polyethylene plastic tube of low ESR background signal. the ESR spectra of the specimen from test and control rats were recorded on an ESR-300 spectrometer (Bruker). The settings for ESR were as follows: power, $10 \mathrm{~mW}$; scan width, $50 \mathrm{G}$; time constant, $40.96 \mathrm{~ms}$; and central field, $3480 \mathrm{G}$, respectively.

\subsection{Extraction and spectra measurements of FA from the bones of the rats}

The cleaned and dried bone was minced and ground. The powder was mixed with $2 \mathrm{ml} 3 \mathrm{~mol} / 1$ $\mathrm{HCl}$ and the mixture was centrifuged. After centrifugation, the precipitate was dissolved in 0.1 $\mathrm{mol} / \mathrm{l} \mathrm{NaOH}$ and centrifuged. The supernatant

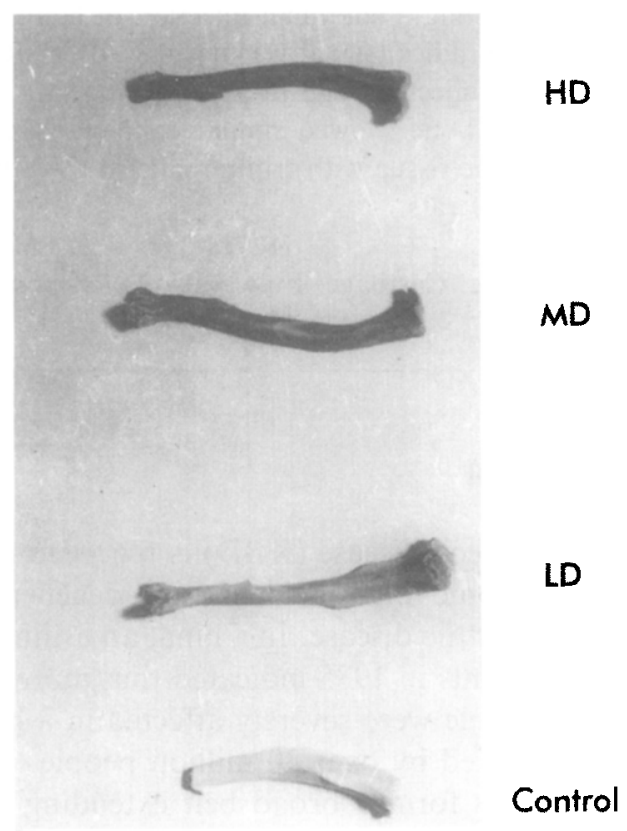

Fig. 1. Photo of the bone of the rats following administration of peat FA at day 130. The injected doses are: HD, 150 $\mathrm{mg} / \mathrm{kg} / \mathrm{day}$ : MD, $100 \mathrm{mg} / \mathrm{kg} / \mathrm{day} ; \mathrm{LD} .50 \mathrm{mg} / \mathrm{kg} / \mathrm{day}$; and CK. control 


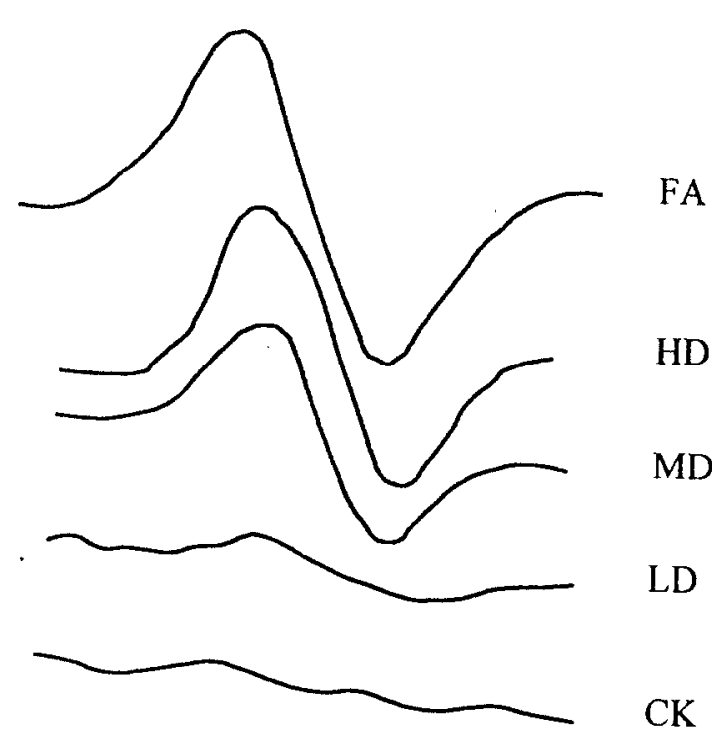

Fig. 2. ESR spectra of the bone specimens of the rats following administration of peat FA. FA: peat FA sample; HD: 150 $\mathrm{mg} / \mathrm{kg} / \mathrm{day}$ : MD: $100 \mathrm{mg} / \mathrm{kg} / \mathrm{day} ; \mathrm{LD}: 50 \mathrm{mg} / \mathrm{kg} / \mathrm{day}$; and $\mathrm{CK}$ : control.

was collected and adjusted to alkaline conditions by using $0.1 \mathrm{~mol} / 1 \mathrm{NaOH}$ to precipitate $\mathrm{Ca}_{3}\left(\mathrm{PO}_{4}\right)_{2}$ and the yellow substances. The precipitate was dissolved in $1 \mathrm{~mol} / 1 \mathrm{HCl}$, brought to $\mathrm{pH} 4.5$ by $0.1 \mathrm{~mol} / 1 \mathrm{NaOH}$, and added with $0.3 \mathrm{ml}$ of 0.1 molil EDTA. The same treatment procedure was performed for the control. The ultra violet spectra (UV) and fluorescence spectra (FL) of the test solutions and FA solution, which was used for injection, were recorded on a UV-3000 spectrophotometer (Shimadzu, Japan) and RF-520 dual beam different fluorophotometer (Shimadzu, Japan). The molecular weight of these two solutions was estimated by gel permeation chromatography (GPC) using Model-0210 HPLC (Waters) and RF-535 fluorophotometer (Shimadzu, Japan) as a detector.

\subsection{Animal testing with tritium labeled $F A$}

FA was extracted from soil of the KaschinBeck disease region (Aba county, Sichuan Province, China) according to an extraction method proposed by the International Humic Substances Society (IHSS, Schnitzer and Preston,
1986). After purification, the FA was tritium labeled using the catalytic isotope exchange method. The labeling was carried out at the Institute of Atomic Energy Research, Chinese Academy of Sciences. The labeled product was washed repeatedly to remove the exchangeable tritium and the final radioactivity was $2 \times 37$ $\mathrm{MBq} / \mathrm{ml}(2 \mathrm{mCi} / \mathrm{ml})$. To each Wistar rat $(145-180$ $\mathrm{g}$, feed with commercial diet), $1.5 \mathrm{ml}$ of solution containing $10 \mathrm{mg}$ FA and $20 \mu \mathrm{Ci}{ }^{3} \mathrm{H}-\mathrm{FA}$ was orally administrated each other day for a total of 10 administrations. For the control group, physiological saline was used. After administration, 10 rats of each were sacrificed at 10,20,30 and 40 days, respectively. The sacrificed rats were immediately dissected to obtain the heart, liver, spleen, lung, kidney, thymus, brain, muscle, skin and hair, bone, and joint cartilage, which were wiped

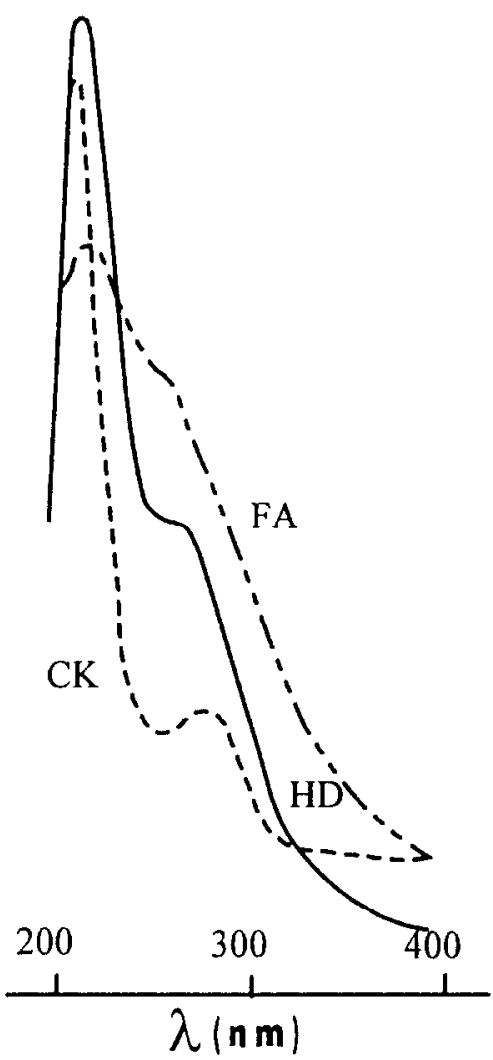

Fig. 3. UV spectra of the extracts of bone specimens of the rats and the solution used for administration. FA: peat sample; HD: extract of the bone injected with $150 \mathrm{mg} / \mathrm{kg} /$ day; $\mathrm{CK}$ : extract of the bone of control. 
dry with filter paper and weighed. The radioactivity was measured for different organs after digestion.

\subsection{In-vitro ${ }^{3} \mathrm{H}-\mathrm{FA}$ incorporation experiment}

Chicken embryo were incubated for 12 days at $37^{\circ} \mathrm{C}$. The two limbs were separated in Hank's solution. After removing muscle tissue, cartilage was separated and digested in trypsin (Sigma) solution for $20 \mathrm{~min}$, then washed with Hank's solution and digested repeatedly. The cartilage was minced to $1 \mathrm{~mm}^{3}$ and washed using 1640 (Sigma) culture solution containing fresh fetal calf serum (FCS, from Beijing Medicine University). The pieces of cartilage tissues for each pot in a block of 24 pots were cultured in 1640 medium and maintained in an incubator at $37^{\circ} \mathrm{C}$. The medium was changed every $24 \mathrm{~h}$. After two days of incubation, the medium was changed to 1640 without FCS and with ${ }^{3} \mathrm{H}-\mathrm{FA}$. The cartilage tissues were collected and washed six times using physiological saline at $12,24,48,96 \mathrm{~h}$, and 7 and 10 days, respectively. The tissue was dried with filter paper and weighed.

\subsection{Digestion of tissue and determination of tritium radioactivity}

Specimen $(100 \mathrm{mg})$ was digested in a mixture of $0.2 \mathrm{ml}$ concentrated $\mathrm{HClO}_{4}$ and $0.4 \mathrm{ml} \mathrm{H}_{2} \mathrm{O}_{2}$ at $70-80^{\circ} \mathrm{C}$ for $30-60 \mathrm{~min}$ when the solution bccame colorless and transparent. The solution was transferred into scintillation bottles. To the bottles, $4 \mathrm{ml}$ glycol monoethyl ether and $8 \mathrm{ml} 0.6 \%$ biphenyl oxazole (BPO, Merck) in methyl benzene scintillation solution was added and mixed thoroughly. The bottle was stood overnight and the scintillation was counted for $1 \mathrm{~min}$ on a LS 6000 TA scintillation counter (Beckman, USA).

\section{Results and discussion}

\subsection{Morphology and spectra verification}

With the injection of peat FA, the color of the rats' bones became yellow or brown in relation to

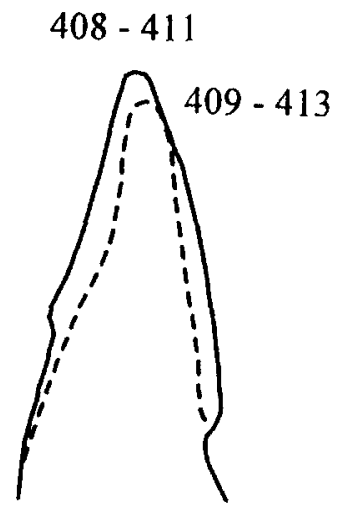

(a)

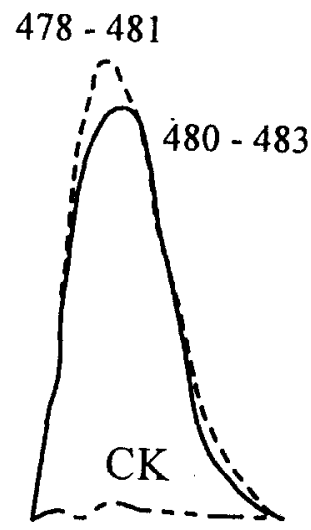

(b)
Fig. 4. Fluorescence spectra of the extracts of bone specimens of the rats and the solution used for administration. --...: FA sample; - . : extract of the bone injected with $150 \mathrm{mg} / \mathrm{kg} / \mathrm{day}$; extract of the bone from the control.

dose. Fig. 1 illustrates limbs from the control and experimental rats injected with different dosage of FA, the bones injected with FA are colored and the depth of color varies with the FA dose injected.

The ESR spectra of the limbs were recorded, as shown in Fig. 2. It showed that the intensity of the ESR signal increases with increasing dose of FA. FA contains quinone and semiquinone structure with a $g$ factor of 2.0034 on ESR spectra. From Fig. 2, the spectra of extracts from limb bones of the rats injected FA were similar to that of FA solution used for injection. Higher ESR signals were observed in FA-injected groups when compared to the control group. The intensity of the signal was positively correlated with FA concentration used for the injection.

The $\mathrm{HCl}-\mathrm{NaOH}$ extracts of bone specimen were characterized by UV, FL, and GPC, as shown in Figs. 3-5, respectively. For comparison, a solution of peat FA was also recorded. The major spectral characteristics of the extractable materials from bones of the experimental rats were similar to the sample of peat FA.

The results of animal tests provide further experimental evidence for a metabolic pathway and incorporation of FA into organs and tissues of the rat. The distribution of ${ }^{3} \mathrm{H}$-labeled $\mathrm{FA}$ in 

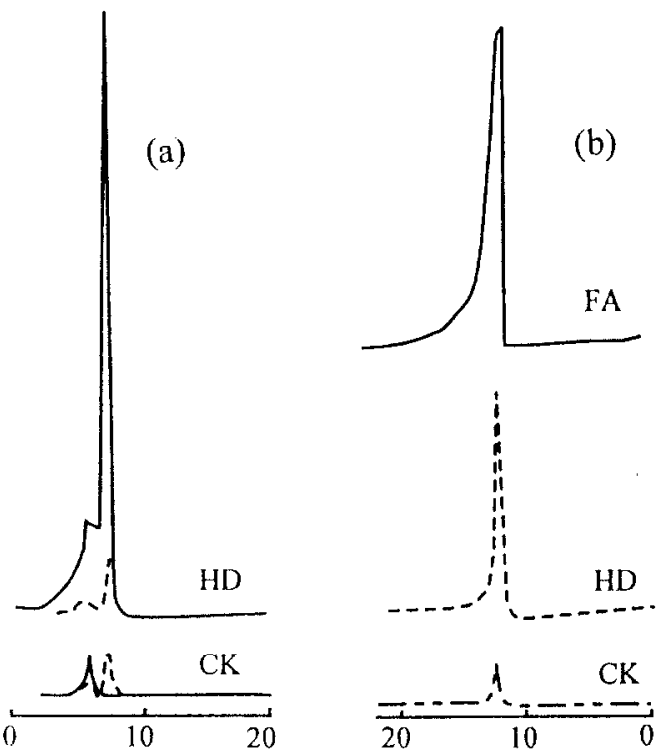

\section{Retention time (min)}

Fig. 5. Gel permeation chromatography of the extracts of bone specimens of the rats and the solution used for admnistration. FA: peat FA sample; HD: extract of the bone injected with $150 \mathrm{mg} / \mathrm{kg} /$ day; $\mathrm{CK}$ : extract of the bone from the control. different organs of the rats is shown in Fig. 6, the FA was distributed in all organs of the rats and the incorporation is not specific. The distribution of radioactivity in different organs follows the order of kidney $>$ liver $>$ spleen $>$ bone $>$ cartilage $>$ skin and hair $\sim$ muscle $\sim$ lung $\sim$ thymus $\sim$ heart $\sim$ brain. The main metabolic organs for FA in rats are liver and kidney, where the radioactivity is higher and the turnover time is shorter than that in other organs. In bone and cartilage, because the metabolism rate of FA is relative slow, these tissues reflect accumulation phenomena. The incorporation of ${ }^{3} \mathrm{H}$-labeled $\mathrm{FA}$ into in-vitro cultured cartilage tissue is shown in Fig. 7 which illustrates that the accumulation of ${ }^{3} \mathrm{H}-\mathrm{FA}$ in cartilage tissue and the radioactivity increases with incubation time. This is a direct testimony that FA does accumulate in the target tissue of KBD.

With experimental evidence of an incorporation of ${ }^{3} \mathrm{H}-\mathrm{FA}$ into in-vivo and in-vitro cultured cartilage, accumulation of ${ }^{3} \mathrm{H}-\mathrm{FA}$ into bone and cartilage of the rats, we conclude that labeled FA attach to and deposit in the target tissue of KBD.

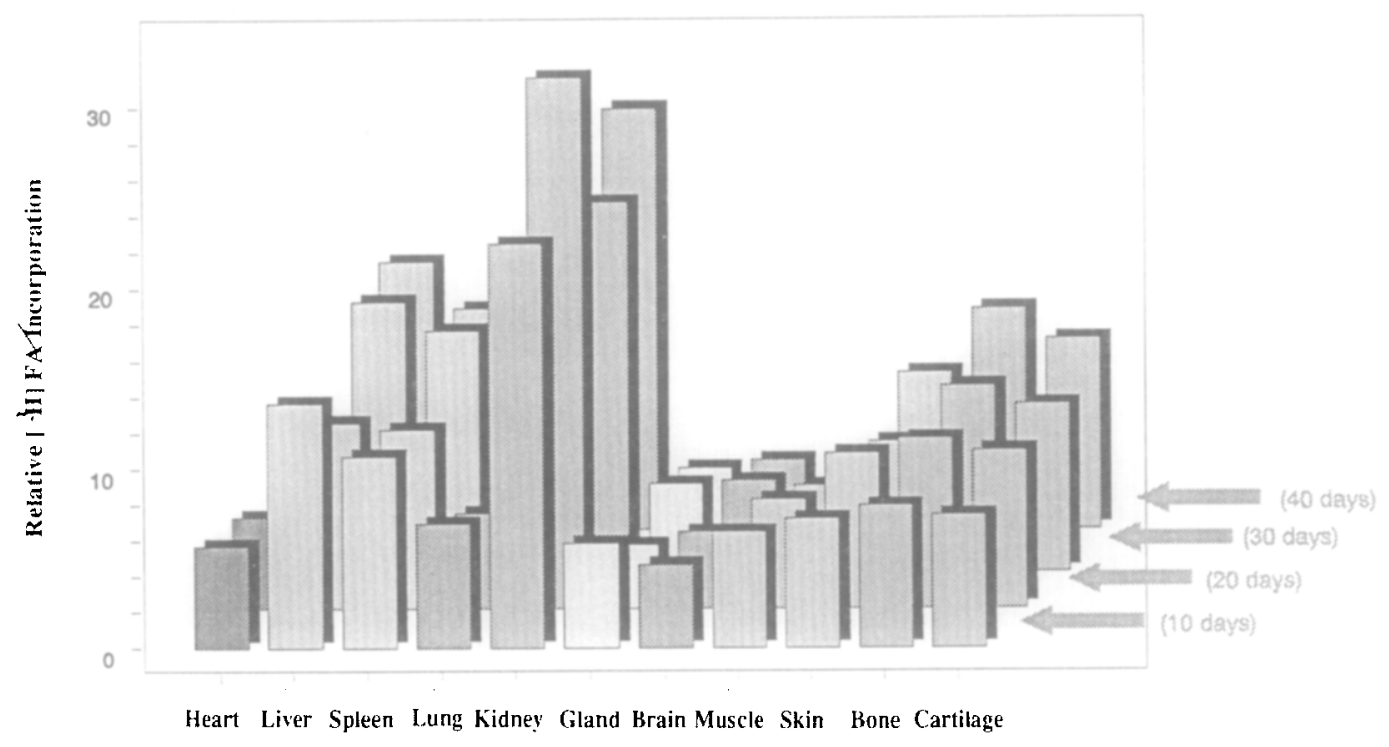

Fig. 6. Time dependant radioactivity of ${ }^{3} \mathrm{H}-\mathrm{FA}$ in different organs and tissues of the ras in in-vivo experiments. 


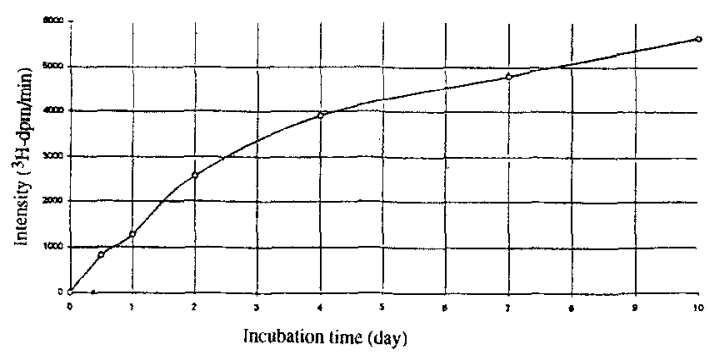

Fig. 7. Time dependent radioactivity of ${ }^{3} \mathrm{H}-\mathrm{FA}$ in chicken embroylic cartilage tissue in in-vitro experiments.

Combined with the identification of UV, FL and GPC spectra of extracts of the bone of the rat and solution of peat FA for injection, we conclude that FA is one of the pathogenic factors of $\mathrm{KBD}$.

\section{Acknowledgements}

This work is supported by the China National Sciences Foundation.

\section{References}

Aiken, G.G., 1985. In: G.R. Aiken and D.M. McKnight et al. (Eds.), Humic Substances in Soil, Sediment and Water. Wiley, New York, pp. 363-385.

Feng, L.F. and W.Zh. Wang, 1990. Environmental Life Elements and Health, Science Press, Beijing, p. 301.

Huang, T.S., F.J. Lu and I.J. Chopra, 1993. Environ. Toxicol. Chem., 12: 1267-1271.

Lu, F.J., S.C. Tsai and J.Y. Chen, 1980. J. Chin. Biochem. Soc., 9: 49-54.

Peng, A., Z.J. Wang, W.H. Wang and C.L. Yang, 1991, J. Environ. Sci. (China), 3 (4): 5-14.

Schnitzer, M. and C.M. Preston, 1986. Soil Sci. Soc. Am. J., 50: $326-331$ 